\title{
On the role of viruses in the evolution of immune responses
}

\author{
Zinkernagel, R M ; Hengartner, H ; Stitz, L
}

\begin{abstract}
Mutual influences of viruses on the immune system and vice versa which lead to a biological balance are considered, with clinical and/or experimental findings. Numbers and turnover of immune cells can be correlated with numbers and growth rate of viruses; certain viruses apparently have adapted to systemic or local immune effector mechanisms. The biological balance of viruses and immune system guarentees overall protection of both host and parasite. It alos may lead to conditions where immune protective mechanisms causes cell and tissue damage leading to disease. Immunologically mediated disease may be influenced by immune regulation via HLA antigen and may therefore explain HLAdisease associations. Finally, the different specificities of antibodies vs. T Cells and the differing kinetics of their immunological memory are outlined and correlated with immune escape, immune protection and the resulting possible evolutionary pressures on viruses
\end{abstract}

DOI: https://doi.org/10.1093/oxfordjournals.bmb.a072033

Posted at the Zurich Open Repository and Archive, University of Zurich

ZORA URL: https://doi.org/10.5167/uzh-155168

Journal Article

Published Version

Originally published at:

Zinkernagel, R M; Hengartner, H; Stitz, L (1985). On the role of viruses in the evolution of immune responses. British Medical Bulletin, 41(1):92-97.

DOI: https://doi.org/10.1093/oxfordjournals.bmb.a072033 


\section{ON THE ROLE OF VIRUSES IN THE EVOLUTION OF IMMUNE RESPONSES}

\section{R M Zinkernagel}

\section{H Hengartner}

Institute for Pathology

University Hospital, Zürich

\section{Stitz}

Institute for Virology

University of Giessen, FRG

Mutual influences of viruses on the immune system and vice verse which lead to a biological balance are considered, with clinical end/or experimental findings. Numbers and turnover of immune cells can be correlated with numbers and growth rate of viruses; certain viruses apparently have adapted to systemic or local immune effector mechanisms. The biological balance of viruses and immune system guarantees overall protection of both host and parasite. It also may lead to conditions where immune protective mechanisms cause cell and tissue damage leading to disease. Immunologically mediated disease may be influenced by immune regulation via HLA antigen and may therefore explain HLA-disease associations. Finally, the different specificities of antibodies vs. T cells and the differing kinetics of their immunological memory are outlined and correlated with immune escape, immune protection and the resulting possible evolutionary pressures on viruses.

Although immunological mechanisms are only one part of a vertebrate host's defence mechanism, they undoubtedly play an important role in limiting infectious disease. Immunodeficient humans or mice usually succumb to infectious disease and not to tumours or degenerative disease. T cell-deficient patients die from viral infections, whereas agammaglobulinaemic children survive many viral infections well but succumb to bacterial infections (for reviews see refs. 1-5). These experiments of Nature clearly indicate that the two main parts of the immune system, humoral and cell-mediated, cover different areas of infectious diseases. Extracellular parasites (mainly bacteria) are checked immunologically by antibodies and complement factors, whereas intracellular parasites (viruses, facultative intracellular bacteria) are controlled mainly by $\mathrm{T}$ cells and cell-mediated immunity.

The host-virus relationship depends on a variety of factors which lead to a well balanced evolutionary equilibrium between infectious agents and hosts. Factors influencing viruses include the degree of cytopathogenicity, organ or cell tropism, susceptibility to immune modulation, to interferon, or to concurrent infections; factors determined by the host are the degree of immunocompetence, immune response regulation, natural resistance factors, genetic factors etc. Some viruses, such as pox and influenza, are highly cytopathic and therefore threaten the life of their host much more than non- or poorly cytolytic viruses (such as human bepatitıs $\mathrm{B}$ virus, or lymphocytic choriomeningitis virus (LCMV) in mice). It is obvious that the more destructive for cells a virus is, the greater is the need for rapid and efficient elimination by the host. The immune system, as we know it in higher vertebrates today, must therefore offer otpimal defence (immune protection) against cytopathic viruses. However, such an efficient approach may have disadvantages when dealing with non-lytic viruses; these may cause immunologically mediated damage of cells and organs, i.e., immunopathology.

\section{Some General Considerations}

\section{Virus Infection and Immunity}

Viruses infect higher vertebrates via skin, mucous membranes or enter the blood directly (via insect bites). After replication, virus reaches local lymph nodes where it replicates further and then spreads systemically via blood to secondary organs. In the case of poxvirus infection or measles, these two phases each take about 4-7 days. Replication time, kinetics of induction of viral antigen on the cell surface, extent of cell destruction caused by the virus etc, vary from one virus to another. All viruses usually give the immune system a signal, either on the surface of infected cells or on antigen-presenting cells (which take up viral antigens produced in surplus or are themselves infected). Infection with some viruses does not lead to induction of virus-specific antigens, while, in other cells, such induction may occur but not be recognized. In these cases, virus may replicate uninhibited before it is recognized immunologically, thus giving the virus a disasterous advantage. Usually $T$ cell-mediated immunity is measurable systemically within 4-6 days after general spread of virus, at about the time when viral titres begin to decrease. In contrast, antibodies become detectable only later, particularly the biologically relevant neutralizing antibodies which are often first measurable days or weeks after virus has disappeared. Antiviral antibodies against various structural and non-structural viral antigens are often detected early, but their antiviral role is unclear.

\section{Number Games}

Infectious agents, particularly viruses, have one advantage over vertebrate hosts : their number. They usually multiply rapidly (e.g. viruses, bacteria) and therefore mutations may arise within short periods of time. Viruses multiply 100 to 10000 times within one infectious cycle (6-24 hours) and bacteria usually divide every $30-$ 60 minutes. This is to be compared with the multiplication or generation times of mice or humans which are orders of magnitude longer, i.e. 3-5 months or 13-25 years respectively.

From this numbers point of view, lymphocytes are the means to render this situation more balanced biologically. Lymphocytes divide every 12-24 hours, and there are some $10^{9}$ in mice or about $10^{12}$ in humans. Their great numbers and rapid replication times allow for mutational adaptation and therefore give appropriate flexibility for immune defence against changing infectious agents.

Therefore, one can explain why the immune system is built of single mobile cells, in great numbers with relatively short half lives and great proliferative potential; such a system is adequate to 
enable long lived hosts to deal efficiently with the many different and highly mutating viruses and bacteria.

\section{Immune Geography}

Viruses differ with regard to cytopathic effects, tropism, replication, interferon induction but also with respect to host range, or the portal of entry into the host. Here we consider two aspects in particular: first, which cells must be infected so that an immune response is triggered and second, what is the role of the entry site of a virus into the host in determining immunologically mediated recovery from virus infections or prevention of reinfection.

Experiments with model systems have clearly shown that an immune response is triggered only if antigen is presented properly by antigen-presenting cells. Rabies virus may serve as an example: it may hide in neurons for a considerable length of time before cytopathic effects destroy cells and viral antigens are set free and are taken up by antigen-presenting cells. ${ }^{8}$ Because of these sometimes rather long incubation periods, early administration of rabies vaccines circumvents the effective seclusion of rabies virus in neurons; i.c., inactivated rabies virus is most probably readily taken up by antigen-presenting cells and this should induce an immune response. This could explain why postexposure vaccination works (sometimes) to prevent or ameliorate rabies. In most other infections, incubation periods are probably too short to make postexposure vaccination an effective means of accelerating immune defence.

There is no doubt that induction of $\mathrm{T}$ cell-mediated immunity depends on viral antigen being presented properly to lymphocytes. In fact, very early after infection most viruses infect tissue macrophages (Langerhans, Kupffer or dendritic cells) as well as macrophages in lymph nodes and spleen. ${ }^{8.9}$ There is no evidence so far that the $T$ cell response against virus entering via skin or mucosal surfaces, or entering directly into the blood differs with respect to specificity, class of $T$ cells (helper, cytotoxic) induced, or with respect to recirculation patterns of $T$ cells. As will be pointed out, this contrasts with the distinct immune geography of antibodies. ${ }^{10}$ Very little is known about the survival time of effector $T$ cells and their distribution or recirculation. Cytotoxic $T$ cell responses fade away extremely rapidly a few days after virus has been eliminated from the organism;3,11 nevertheless, the conventional view is that delayed-type hypersensitivity (DTH) mediated by $T$ cells is long-lived and can be elicited for many years. ${ }^{1.2 .5,12}$ However, this notion may be misleading for two reasons. First, there is some, although still weak, evidence that antibodies may be involved in DTH ${ }^{13}$ and second, there are only few infectious disease models where DTH can be shown in humans (e.g. in tuberculosis, leprosy, sarcoidosis, etc.) or in mice, and where there is no reasonable evidence that either infectious agents and/or their antigens persists for a long time. ${ }^{12}$ Such concomitant immunity exists where established parasites survive in the presence of an immune response whereas newly spreading or freshly entering bacteria of the same type are eliminated efficiently. ${ }^{2}$

$B$ cells and antibodies, in contrast to T cells, exhibit a rather marked linkage to the location within the host: IgA on mucous membranes, IgE in the gut and the skin, IgG and IgM in the circulation. Antibody responses usually appear more slowly than $T$ cell immunity; this is probably due largely to the fact that $T$ helper cells must first be induced before efficient $B$ cell triggering occurs. ${ }^{14}$ Also, in contrast to $T$ cells, antibody responses are longlasting and memory persists often for many years.
During a virus infection, $T$ cells are normally triggered rapidly and decline soon after virus elimination; $T$ cell responses are shortlived, are not easily recalled (see later) and often seem to show a somewhat broader specificity than B cell responses. ${ }^{14,15}$ In the Listeria monocytogenes model in mice and rats, memory $T$ cells apparently do not recirculate very efficiently when measured some weeks after infection. ${ }^{16,17}$ Thus, memory $T$ cells, if they exist at all, seem to be sessile ${ }^{17}$ and therefore probably not very efficient in preventing reinfections at other locations in the host, except perhaps when the agent enters the spleen.

\section{Immune Protection Versas Immumopathology}

The immune system is made to eliminate acute, cytopathic viruses or intracellular bacteria. In general, it fulfils this task very well indeed. Once the infectious agents and their antigens are eliminated, the immune responses fade away quickly. Thus, for acute infectious agents, one does not have to evoke sophisticated suppressor cells or cellular circuits to regulate an immune response. ${ }^{18}$ Rapid increase in the concentration of foreign antigen on specialized cells ${ }^{6}$ (macrophages, antigen presenting cells, dendritic follicular cells) is stimulus enough to provoke a $T$ cell- or $B$ cell-mediated immune response; mere elimination of such antigens is enough to stop it.

However, some viruses-maybe most-are not acute cytopathic agents; therefore such viruses (or bacteria) are not evolutionarily selective and infected hosts do survive independent of the immune response. Such agents may be able to infect immune-deficient hosts or may survive in any host for a long time without causing disease or death. Hepatitis B virus infections or leprosy in humans or LCMV infection in mice may serve as examples. The majority $(>90 \%$ ) of infectious people generate a potent immune response, eliminate infectious agents efficiently and rapidly and therefore show no or few symptoms. The few patients lacking an effective immune response completely become virus carriers or carriers of leprospy bacilli ${ }^{19,29}$ (hepatitis B virus carriers, polar lepromatous leprosy patients). In a few percent of infected patients, immune effector mechanisms cannot quite get rid of infectious agents. The persistant conflict between immunity and infection results in immunopathological damage as, for example, in agressive hepatitis or tuberculoid leprosy. Whenever foreign antigens persist, immunopathology develops, be it via $\mathrm{T}$ cell-mediated effector mechanisms, or via antibodies forming immune complexes. ${ }^{21} \mathrm{~A}$ common feature of these particular disease states is that the infectious agents are of inherently low pathogenicity with a resulting low mortality rate. Furthermore, the disease may develop slowly without interfering severely with reproduction. Immune response regulation, ${ }^{15,22,28}$ among other parameters, may therefore set the stage for developing disease through immunological damage. Because major transplantation antigens influence immune responsiveness profoundly, disease susceptibility may be associated with certain HLA antigens.

\section{Modulation of the Immune response to an Infections Agent by Concomitant Infections by Other Agents}

The disease and the immune responses induced by one infectious agent may modulate the disease and the immune responses induced by a subsequent infection with a different agent. A classical example is the finding that skin reactions to tuberculin disappear during measles infection. ${ }^{1,5}$ All researchers doing experiments in mice know that 'sick' mice may give very weak immune responses 


\section{THE ROLE OF VIRUSES IN THE EVOLUTION OF IMMUNE RESPONSES $R$ M Zinkernagel et al.}

in vivo or in vitro. Immune-deficient hosts often show an aberrant immune reaction not only because of lack of certain immunological effector mechanisms, but because these deficiencies lead to altered functions of the existing immune apparatus; e.g. thymus-deficient nude mice $(n u / n u)$ possess macrophages that are much more activated than those found in normal mice. ${ }^{29}$ In most examples studied, an existing infectious disease tends to attenuate a second concomitant infection. The pathophysiological mechanisms involved are not well known; lymphokines, interferons, activated macrophages, increased natural killer cell activities etc. may play important roles. ${ }^{30-32}$ However, it has become apparent more recently that infectious agents, particularly viruses, may be responsible for acquired immunodeficiencies. It is far from clear how a lymphotropic or lymphocytopathic virus or the concomitant infection by various viruses together lead to impairment of the immune system. Nevertheless acquired immune deficiency syndrome (AIDS) may well be the result of such concurrent infections rather than of a human $\mathrm{T}$ leukemia virus (THLV) infection alone. ${ }^{33}$

For all we known, these examples of the negative effect of viruses on the immune system may be the exceptions rather than the rule. Understanding the mechanisms underlying these diseases will elucidate important parameters determining the equilibrium between infectious agents and immune responses.

\section{Antiviral Immunity and Polymorphism of Major Transplantation Antigens}

The fact that the major histocompatibility gene complex (MHC) influences immune responsiveness has been known for some time, ${ }^{22-28}$ but there is also substantial evidence for important roles of non-MHC genes in regulating susceptibility to infectious diseases. ${ }^{1-5}$ Historically MHC immune-response (Ir) genes have been studied most extensively with respect to their role in antibody responses:24,28 their regulatory role is antigen-specific, dose dependent, and MHC haplotype (H-2I region in mice, HLA-D/DR in humans)-dependent. Although the responsiveness phenotype is dominant, non-responder $T$ cell-B cell interactions cannot be rescued. Ir genes probably exert their effect on $T$ helper cells either at the level of the receptor repertoire or at the level of antigen presentation. Similar effects have been mapped to H-2I and HLA$\mathrm{D} / \mathrm{DR}$ for $\mathrm{T}$ cell responses measured by proliferation or DTH. ${ }^{27}$

Ir gene-dependent regulation of the generation of cytotoxic effector $T$ cells operates in responses against chemically modified cells and minor histocompatibility antigen, as well as against viral antigens. ${ }^{15}$ These latter types of Ir genes act antigen-specifically (to a certain degree), they depend on the MHC haplotype and map to $\mathrm{K}$ or $\mathrm{D}$ or HLA-A or $\mathrm{B},{ }^{34,1}$ s i.e., to the same MHC regions that code for the restricting self-histocompatibility antigen (self-H) of cytotoxic $T$ cells. Again, non-responsiveness linked to a nonresponder $\mathrm{H}-2 \mathrm{~K}$ or D or HLA-A or B cell cannot be rescued and has a dominant character. ${ }^{15}$

Thus, MHC-linked Ir genes that regulate responsiveness of $\mathrm{T}$ cells and the genes coding for the restricting self-H determinant are apparently identical. This conclusion is strengthened by the finding that $T$ cell specificity for self is selected in the thymus and by the thymic $\mathrm{MHC}$, as is the Ir-phenotype of maturing T cells. ${ }^{15}$ Therefore, selection of the specificity for thymic self-H also automatically determines the Ir phenotype. In summary Ir-gene phenomena are a direct consequence of $T$ cells being MHCrestricted.

\section{Why MHC Polymorphism?}

The extreme polymorphism of MHC products has been explained in many different ways. Most hypotheses assume that the interest of viruses in K, D products of $\mathrm{H}-2$ or A, B of HLA may stem from the fact that the latter have evolved from cell and organ growth-regulating differentiation antigens. The theories differ mainly in that some imply immunologic reasons for polymorphism and others do not. ${ }^{22-24,35}$ Arguments that polymorphism is not related to immunity are that polymorphism may serve as a marker system of individuality that prevents mutual fusion or parasitism between members of the same or other species. When coupled with the immune system, polymorphism may prevent the spread of infectious tumours. Alternatively, polymorphism may have developed as a pure accident of nature and is maintained only because relatively closely linked loci are polymorphic (e.g. $\mathrm{T} / \mathrm{t}$ in the mouse). A different hypothesis is that, since many viruses replicate best in multiplying cells, interaction of a virus with cellsurface antigens involved in cell differentiation and proliferation may influence susceptibility to infections. None of these mechanisms can be disproved, and some may in fact operate in parallel. As detailed elsewhere in this volume and in preceding sections here, the most compelling idea is that MHC polymorphism and MHC-restricted, cell-mediated immunity to intracellular parasites are intimately linked. Therefore, because $\mathrm{T}$ cell effector function is determined by self-MHC products, the size of the species' T-cell receptor repertoire for foreign antigens is directly dependent on the polymorphism of the MHC products. ${ }^{15.22-28.35}$

Mice infected with the enveloped viruses tested so far generate strong cytotoxic $T$ cell responses. If this immune activity is separated into an $\mathrm{H}-2 \mathrm{~K}$ and $\mathrm{H}-2 \mathrm{D}$-restricted response, and one tests $T$ cell activity against self- $K$ plus virus and against self- $D$ plus virus separately, great differences are detected. For example, $\mathbf{K}^{\mathbf{k}}$ is associated with very high response to a poxvirus but rather weak response to lymphocytic choriomeningitis virus. In contrast, $D^{k}$ is associated with high response to LCMV, but no measurable response to the poxvirus. Similar examples may be found for HLA-A,B restricted T cell function in human ${ }^{23}$ or for HLA-DR or H-2I restricted T cells. ${ }^{27}$

Although the following discussion deals mainly with expression of virus-specific cytotoxic $T$ cells, it is understood that I-restricted $T$ cell responsiveness parallels the following example: cytotoxic $T$ cells are essential for survival and if mice possessed only one single self-H marker as a receptor for lytic signals delivered by specific $\mathrm{T}$ cells, for example $D^{k}$, the consequences would be disastrous for the species' survival. The first poxvirus pandemic would eliminate all mice. Polymorphism of self-H alone would reduce this chance substantially, but not eliminate it entirely, since deaths would accumulate as one after another of many possible highly mutating viruses attack. Only duplication of self- $\mathrm{H}$ together with polymorphism deal effectively with the problem. It would be difficult for any virus to mutate in such a way as to mimic two or four self- $\mathrm{H}$ markers at the same time or otherwise escape MHC-restricted immune surveillance. ${ }^{35}$

This concept implies that MHC products functioning as we know them today in $\mathrm{T}$ cell-mediated immunity, fulfilled other, related functions earlier during phylogeny. In fact, duplication of these original MHC loci may have allowed some of them to be sequestered fucntionally to co-evolve with $T$ cell immunity and become highly polymorphic. Others may have remained with original functions as markers for cell interactions, cell differentiation, organ formation, etc. Some of the many MHC-linked $T$, $Q a$, etc. loci may well represent such examples. 
In essence, we believe MHC polymorphism has developed under selective pressure by intracellular parasites. This conclusion is based on the fact that MHC-coded self-H defines the effector function of $\mathrm{T}$ cells, influencing $\mathrm{T}$ cell immune responsiveness because $\mathrm{T}$ cells are $\mathrm{MHC}$-restricted.

MHC-Associated Diseases are Medlated by Immunopathological Processes Exerted by MHC-Restrleted T Cells: A Speculation

Associations between MHC and disease are not found for acute infectious diseases but for chronic diseases with an aura of 'autoaggressiveness'. ${ }^{36,15} \mathrm{~T}$ cell-mediated immunity is intimately tied to MHC products as outlined in the preceding section.

Recovery from infectious diseases caused by viruses can be viewed as resulting from the balance between the viruses' ability to destroy cells (cytopathic effect) and the T cells' ability to kill cells and destroy tissue (cytotoxicity and/or recruitment of inflammatory cells) to prevent further virus replication. Lymphocytic choriomeningitis in the mouse ${ }^{19}$ and aggressive hepatitis or tuberculoid leprosy ${ }^{20}$ in humans may serve as examples. This balance is obviously influenced on one hand by the infectious agent's cytopathogenicity, tropism, rapidity of spread, generation time, and susceptibility to other factors such as antibody-mediated modulation, and on the other hand by the host's degree of immunocompetence and MHC-linked Ir gene-dependent immune responsiveness. ${ }^{1-5,15}$

The response to the highly cytopathic viruses that most commonly afflict young individuals who are too young to reproduce either leads to elimination of the virus or to death of the host. Therefore, life-threatening infectious intracellular agents to which a population is exposed normally will eliminate low responders. Survivors will consist only of phenotypic high responders, and no association between $\mathrm{MHC}$ and susceptibility to disease are noticeable. The finding that all mice are high responders to pox viruses, LCMV, or parainfluenza virus, three of the most prevalent infectious agent of the species, fits this concept very well indeed.

It appears as if $\mathrm{MHC}$-associated diseases are generally found only in relation to noncytopathic or poorly cytopathic agents that cause chronic infections and do not usually interfere with reproduction. Only these types of infections leave a certain leeway for the balance between immunoprotection versus the damage that is caused either by the infectious agents or, more importantly, by the ensuing immune response. From this point of view, we would consider MHC-associated diseases to be immunopathologically mediated diseases and vice versa. ${ }^{15}$

The association between susceptibility to disease and the MHC may develop depending again on the viruses' characteristics, its susceptibility to immune modulation, and the host's immunocompetence at the time of contact as follows. (a) MHC-linked low responsiveness to a poorly cytopathogenic virus may magnify the spread of virus and the subsequent extensive and chronic cellmediated destruction of host tissue. Here, low-responder MHC alleles are associated with increased susceptibility when compared with high responder $\mathrm{MHC}$ alleles. (b) MHC-linked low responsiveness to a poorly cytopathogenic virus that has already spread widely (under the cover of prenatal immunoincompetence, for example, or because of temporary immune modulation by passive maternal immunity or concurrent infection with other agents) may be associated with decreased susceptibility to disease. The latter example would fit the fact that many MHC disease associations are dominant.

An important characteristic of the association between MHC type and susceptibility to disease is that it involves the entire haplotype configuration rather than single MHC alleles. Perhaps this may be explained by the following proposal: since MHCrestricted $T$ cell activities are interconnected, certain combinations of HLA-A plus HLA-D or HLA-B plus various HLA-D region alleles may influence the overall result of immune responsiveness; e.g. HLA-D-restricted interactions between $T$ helper cells and other lymphocytes or between $T$ cells and macrophages may ultimately generate HLA-A,B-restricted cytotoxic T cells. Alternatively, the inaccuracy of serological MHC typing may render associations more loose than they actually are. Either mechanism may cause linkage with haplotype rather than single loci.

MHC disease association reflects involvement of MHCrestricted $T$ cells in the disease process. What are the consequences of such a proposal? As mentioned above, immunopathology may result from various pathophysiological mechanisms. Therefore, only some individuals may profit from immunosuppressive therapy, although at this time no guidelines are at hand.

The above speculation suggests careful examination of the use of attenuated live vaccines. Such vaccines may shift the balance of the immune response from immune protection towards immunopathological damage. On the other hand, if an infectious agent causes immunopathological disease, an appropriate vaccine should induce efficient elimination of this agent before widespread immunopathologic damage is done. Ultimately, to understand immunopathological disease and its linkage to MHC and to devise treatment, we must have at hand a comprehensive biochemical analysis of $\mathrm{T}$ cell receptors and of the recognized foreign (viral, bacterial) and self determinants. Furthermore we must have a refined knowledge of viral physiology and of the interaction between virus and vertebrate host.

\section{T Cell vs. B Cell Specificity Against Viruses}

The two major pathways of antiviral immunity are cellular and humoral immunity. Cellular immunity is represented mainly by $T$ cells that act locally via contact on a one-to-one cell basis in solid tissue. The humoral immune system is composed mainly of $B$ cells, whose induction depends on $T$ helper cells. The biologically active products of B cells, 'antibodies', multiply the effector units of one B cell more than 10000 times in concentration independent (IgM) or dependent forms ( $\mathrm{IgG}, \mathrm{IgA}, \mathrm{IgE}$ ). Both $\mathrm{T}$ cells and antibodies have in common that uniform and stereotyped mechanisms are focused on to the site where foreign antigen is present. Whereas $T$ cells recognize antigen only when associated with cell surfaces, B cells recognize both cellular and soluble antigens. Induction and triggering of effector mechanisms of $T$ cells is mediated via their specificity for self-MHC products. Antibody effector function is mediated via the $\mathrm{Fc}$ portion, which binds either to $\mathrm{Fc}$ receptors (e.g. IgE on mast cells or basophils) or binds Clq, initiating the complement cascade. Therefore, effector functions triggered via $T$ cell or antibody recognition are similar; both link specificity for foreign antigenic determinants with stereotypic receptors for mediating effector functions. The fact that at least some of the complement components map within the $\mathrm{MHC}$, as do the restriction elements for $T$ cells, is an interesting finding that has evoked speculation, but so far no definite explanations.

A major problem for the purely analytical approach to immunology is that immunological specificity is relative. Specificity of antibodies can be defined adequately by association constants. Although an antibody anti-A may bind to A only 100 to 1000 times better than to $B$, there is usually no dispute that this antibody (particularly in concert with the rest of the complex anti- 


\section{THE ROLE OF VIRUSES IN THE EVOLUTION OF IMMUNE RESPONSES $R$ M Zinkernagel et al.}

A immune response) is specific against A. Since we cannot measure $T$ cell receptor binding quantitatively to any comparable extent, we must be very careful not to overinterpret experimental results with $\mathrm{T}$ cells obtained usually on a simple $+/-$ scale. Besides evidence that $T$ cells may recognize similar or identical antigenic determinants ${ }^{14}$ (particularly on small proteins used often in experimental immunology) to those seen by $B$ cells, there is also data which suggests that $T$ cells recognize different parts of biologically relevant (e.g. viral) antigens than do B cell. ${ }^{37-40}$ The reasons for these differences are still debated and unclear but one way of resolving them would be to show that there were $T$ cell clones that recognize the same determinants on viral haemagglutinins as do B cells. There is evidence, however that cross-reacting $\mathrm{T}$ cell precursors predominate in primary immune responses. ${ }^{37-39}$ To argue that $T$ cells possess a different receptor repertoire and different receptors from $B$ cells is unfounded at the moment, since we have no direct evidence for or against such speculations.

Most likely, however, differing specificities of $T$ and $B$ cells may reflect topographical characteristics of antigens on cell surfaces, where virally induced antigens usually trigger immune responses. Because $T$ cells recognize antigen-X together with self-MHC products, both $\mathrm{X}$ and self-MHC must be fairly close on the cell surface, irrespective of which $\mathrm{T}$ cell receptor model ( 1 or 2 receptor site(s)) is valid. If determinant $\mathrm{X}$ is much more distal from (or too proximal to) the cell surface compared to the relevant self-MHCdeterminant, $X$ will not induce a proper response. Since B cells recognize $X$ independently of self-MHC they may preferentially recognize peripheral determinants on cell surface because of their easy accessibility. Theoretical calculations have been made to evaluate access of antibody to determinants on influenza virus haemagglutinins. The results suggest that antibody may interact with distal (more variable) determinants but not with the (constant) proximal ones of haemagglutinins mainly because of simple space restrictions. ${ }^{41}$ Therefore, one may speculate that the relative topography of viral determinants on both infected cells and on virus particles may directly determine $T$ vs. $B$ immune responses and specificity. Vice versa, the antigenic structure and topography of relevant viral antigens may be moulded by the immune system so as to permit survival of both viruses and vertebrate hosts, ${ }^{39-41}$ an example of which is given in the following section.

A special case is the interdependence of the variation of influenza virus haemagglutinins and neutralizing antibodies produced in the population. Epidemiological and serological evidence in vivo and more recently experiments in vitro suggest that the changing patterns of influenza virus infections are the results of pre-existing antibodies guiding evolution of new influenza virus strains. Elegant reviews have been written and interesting speculations have been presented to explain why influenza viruses change within a serological subtype (e.g. H3) several times by antigenic drift before new haemagglutinin subtypes (e.g. H2) appear epidemiologically, a phenomenon called antigenic shift. ${ }^{39}$ Whereas the former phenomenon is most often caused by mutations, the latter process is due to reassortment of various influenza gene segments of differing origins. Antibodies pre-existing in the population thus influence the selection and the success of one or other new viral haemagglutinins.

\section{T Versus B Cell Memory}

The high degree of non-cross-reactivity of neutralizing antiviral antibody, compared with frequent cross-reactivity of $T$ cell against serologically distinct viruses, is probably the result of evolutionary pressures by the immune system exerted on viruses that depend on haematogenic spread and/or infection of IgA-releasing mucous membranes. This conclusion is based on the following findings. $T$ cells disappear quickly after a primary infection whereas antibodies usually persist at high level; the latter effectively and immediately neutralize reinfecting identical and newly infecting serologically cross-reactive viruses. Within given geographic areas, selection therefore favours serologically non-cross-reactive viruses. ${ }^{39-41}$ Conventionally, immunological memory is accepted to exist at both the $B$ and $T$ cell level. ${ }^{14}$ The concept of vaccination depends entirely on the existence of immunological memory. Nevertheless, it is not quite clear whether B cell memory is kept alive because of (a) persistence of antigen, (b) continuous exposure to cross-reactive 'natural' environmental antigen, or (c) existence of true memory B cells. Evidence for $T$ cell memory has been summarized in various reviews (e.g. ref. 14). Presumed $T$ cell memory has been shown with classical experiments on carrierspecific $T$ cells helping hapten-specific B cells. A common factor in most of these studies is that $\mathrm{T}$ cell memory was studied rather early, i.e., 3-8 weeks after priming. One therefore may argue that $T$ cell memory is more a quantitative than a qualitative change, in that the precursor frequencies of relevant $T$ cells are temporarily increased but that this persists only for a short period of time.

What speaks against the existence and/or the biological relevance of memory $T$ cells? Firstly, $T$ cell responses against viruses $^{11,15}$ or facultative intracellular bacteria ${ }^{16,17}$ are rather short-lived. Anti-viral cytotoxic T lymphocyte (CTL) responses usually peak about $2-3$ days after virus titres are maximal and are not measurable beyond 2-4 days after viral titres have fallen below detection level; the same is true for $T$ cell responses against facultative intracellular bacteria such as $L$. monocytogenes. Secondly if the kinetics of secondary antiviral $T$ cell responses are compared with primary responses, hardly any of the classical parameters of immunological memory are noticeable. There is (a) no appreciable acceleration and (b) no augmentation of the response (increase of affinity cannot be assessed for $T$ cells). Our own experiments (unpublished) have shown this clearly for CTL responses against vaccinia virus, vesicular stomatitis virus and LCMV in mice. If one examines data from the biased point of view presented here, the same interpretation is hidden in studies on T cell responses against arborviruse ${ }^{44}$ influenza virus ${ }^{37,38}$ and $L$. monocytogenes. ${ }^{16,17}$ Thirdly, there is old evidence in the literature that memory and protection against vaccinia virus, which is predominantly $\mathrm{T}$ cell dependent, is rather short-lived. There is, however, no doubt that vaccination with vaccinia does protect against systemic haematogenic spread (as do all vaccinations) because of triggering of neutralizing antibodies.

Admittedly, the speculation presented here does not explain satisfactorily one finding of experimental in-vitro immunology. That is that unprimed lymphocytes carnot be triggered to a measurable antiviral response in vitro, whereas in-vivo primed $T$ cells readily respond to restimulation in vitro. Whether this is due to increased relative precursor frequencies alone or because our culture conditions are still somewhat limiting remains to be evaluated.

The existing cross-reactive $T$ memory cells are apparently rare and they need time for amplification before migrating and functioning at the site of infection. Protection by secondary $\mathrm{T}$ cell responses is speeded up only slightly or not at all as compared to recovery from primary infections, leaving ample time for virus replication. Therefore, recovery from primary infections by viruses (e.g. influenza, reoviruses, VSV, and togaviruses) that are 


\section{THE ROLE OF VIRUSES IN THE EVOLUTION OF IMMUNE RESPONSES $R M$ Zinkernagel et al.}

(a) related and exist in serologically non-cross-reactive subtypes, and (b) induce cross-reactive $T$ cells, may well depend on cellular immunity ( $T$ helper and cytotoxic cells) and later also on humoral immunity. In contrast, protection against reinfection by these viruses may depend mostly on pre-existing local IgA (e.g. influenza, VSV) or systemic IgG antibodies (e.g. togaviruses).

Both the differing specificities of antiviral antibody vs. $T$ cell responses and the differences in B cell memory and persistence of an antibody responses vs. absence of $T$ cell memory may be explained by the balanced interrelationship between infectious agents and veretebrate host. The question why B cell memory apparently exists in contrast to $T$ cell memory is unanswered but puzzling. Is it because antibodies and $B$ cells form a circular system as speculated in the network hypotheses? ${ }^{45.46}$ Alternatively, it is because of the requirement for vertical transmission of antibodies from mother to offspring, via placenta in utero, via milk neonatally or via egg yolk in chickens to provide early protection during ontogeny? In contrast, $T$ cells are the physiologically older part of the immune system, which cannot be transmitted from mother to offspring. Therefore $\mathrm{T}$ cells may have to be maximally and optimally functioning all the time. What good would immunological memory be if the host were killed during a promary infection? Therefore $\mathrm{T}$ cell responses against intracellular parasites must be as efficient and as quick as possible.

The most important practical implications of these arguments, if they prove to be correct, are obvious: antibodies or $T$ cells are efficient only against determinants which they can recognize appropriately for topographical reasons. Thus, protection by antibodies can be provided only against determinants that are properly exposed under physiological conditions. Therefore, only a few of the possible determinants synthesized with conventional protein synthesis or DNA technology will prove helpful and it is not likely that 'new' relevant and protective determinants will be found by these methods. Secondly, protection by vaccination to induce $T$ cell responses is inefficient and very short-lived; biologically important and long-lasting protection is provided only by antibodies and B cell memory of the correct immunoglobulin class and location.

\section{REFERENCES}

1 Mims CA. Pathogenesis of infectious disease. 2nd ed. London: Academic Press, 1982

2 Notkins AL, ed. Viral immunology and immunopathology. New York: Academic Press, 1975

3 Moller $G$, ed. The Immune response to infectious disease. Transplant Rev 1974; 19

4 Moller, G, ed. Specificity of effector T lymphocytes. Transplant Rev $1976 ; 29$

5 Burnet FM. Principles of animal virology. New York: Academic Press, 1955

6 Möller $G$, ed. Accessory cells in the immune response Immunol Rev $1980 ; 53$

7 Allison AC, Engui EM. Langerhans cells in parasitic diseases. In: Torrigianı G, Bell R, eds. immunological recognition and effector mechanisms in infectious disease. Basel: Schwabe \& Co, 1981; 55-67

8 Lafferty KJ, Wootnough J. The origin and mechanisms of the allograft reaction. Immunol Rev 1977; 35: 231-262

9 Mims CA. Aspects of the pathogenesis of virus diseases. Bacteriol Rev $1964 ; 28: 30-71$

10 Tomasi $\mathrm{TB} \mathrm{Jr}$, Bienenstock $\mathrm{J}$. Secretory immunoglobulins. Adv Immunol 1968, 9: 1-63

11 Blanden RV. T cell response to viral and bacterial infection. Transplant Rev 1974; 19: 56-84

12 Wing EJ, Remington JS. Delayed Hypersensitivity and macrophage functions. In: Fudenberg et al, eds. Basic and Clinical Immunology 3rd ed. Los Altos CA: Lange, 1980: 129-143

13 Crowle AJ. Delayed hypersensitivity in the mouse. Adv Immunol $1975 ; 20: 197-264$

$14 \mathrm{Katz} \mathrm{DH}$. Lymphocyte differentiation, recognition and regulation. New York: Academic Press, 1977

15 Zinkernagel RM, Doherty PC. MHC restricted cytotoxic T cells: studies on the biological role of polymorphic major transplantation antigens determining T-cel restriction $\longrightarrow$ pecificity, function and responsiveness. Adv Immunol 1979; 27: 51-177

16 Mackaness GB. The influence of immunologically committed lymphoid cells on macrophage activity in vivo. J Exp Med 1969; 129: 973-992

17 Jungi TW. Immunological memory to Luteria monocytogenes in Rodents. J Reticuloendothel Soc 1980; 28: 405-417

18 Cantor $\mathbf{H}$, Gershon RK. Immunological circuitis : cellular composition Fed Proc 1979; 38: 2058-2064

19 Hotchin J. Persistent and stow virus infections. Monographs in Virology $1971 ; 3: 1-211$

20 Godal T, Myklestad B, Samuel DR, Myrvang B. Characterization of the cellular immune defect in lepromatous leprosy: a specific lack of circulating Mycobacterium leprae-reactive lymphocytes. Clin Exp Immunol 1971: $9: 821-831$

21 Theofilopoulos AN, Dixon FJ. The biology and detection of immune complexes. Adv Immunol 1979; 28:89-220

22 Snell GD. The H-2 locus of the mouse: observations and speculations concerning its comparative genetics and its polymorphism. Folı Biol (Praha) 1968; 14: 335-365
23 Bodmer WF. Evolutionary significance of the HL-A system. Nature (London) 1972; 273: 139-145

24 Benecerraf B, McDevitt HO Histocompatibility-linked immune response genes. Science 1972; 175: 273-279

25 Burnet FM. Multiple polymorphism in relation to histocompatibility antigens. Nature (London) $1973 ; 245: 359-361$

26 McMichael AJ, Ting A, Zweerink HJ, Askonas BA. HLA restriction of cell mediated lysis of influenza virus-infected human cells. Nature (London) 1977; 270: 524-526

27 Thorsby E. Biological function of HLA. Tissue Antigens 1978, 11: 312-329

28 Möller G, ed. Ir genes and T lymphocytes. Immunol Rev 1978, 38

29 Zinkernagel RM, Blanden KV. Macrophage actıvation in mice lacking thymus-derived (T) cells. Experientia 1975; 31: 591-593

30 Bloom BR, Jimenez, L Marcus PI. A plaque assay for enumeratıng antigen-sensitive cells in delayed-type hypersensitivity. J Exp Med $1970 ; 132: 16-24$

31 Bloom BR, Rager-Zisman B. Cell-mediated immunity in viral infections. In Notkjns AL, ed. Viral Immunology and immunopathology New York: Academic Press, 1975; 113-136

32 Brenan $\mathbf{M}$, Zinkernagel $\mathbf{R M}$. Influence of one virus infection on a second concurrent primary in vivo Infect Immunol $1978 ; 41: 470-475$

33 Weiss R. Retroviruses linked with AIDS. Nature (London) 1984; 309: 12-13

34 Biddison WE, Payne SM, Shearer GM, Shaw S. Human cytotoxic T cell responses to trinitrophenyl hapten and influenza virus. J Exp Med $1980 ; 152: 204 \mathrm{~S}-217 \mathrm{~S}$

35 Doherty PC, Zinkernagel RM A biological role for the major histocompatibility antigens. Lancet $1975 ; 1: 1406-1409$

36 Dausset J, Svejgaard A, eds. HLA and disease. Copenhagen: Munksgaard, 1977

37 Effros RB, Doherty PC, Gerhard W, Bennink J. Generation of both cross-reactive and virus-specific $T$ cell populations after immunization with serologically distinct influenza A viruses. J Exp Med 1977, 145: $557-568$

38 Braciale TJ, Ada GL, Yap KL. Functional and structural consideration in the recognition of virus-infected cells by cytotoxic $T$ lymphocytes. Contemp Top Mol Immunol. 1978; 7: 319

39 Fazekas de $\mathrm{St}$ Groth $\mathrm{S}$. The joint evolution of antigens and antibodies. In: Steinberg Ch M, Leflovits I, eds. The immune system. Basel: Karger, 1981; 1: 155-168

40 Zinkernagel RM, Rosenthal KL. Experiments and speculation on antiviral specficity of $T$ and B cells. Immunol Rev 1981, 58:131-155

41 Fazekas de St Groth S. Cross recognition and cross reactivity. Symp Quant Biol Cold Spring Harbor 1967; 32. 525-536

42 Wolcott JA, Wust CJ, Brown A. Immunization with one alphavirus cross-primes celular and humoral immune responses to a second alphavirus. J Immunol 1982; 129: 1267-1271

43 Lindenmann J. Speculations on tdiotypes and homobodies. Ann Immunol (Paris) 1973; 124C: 171-184

44 Jerne NK. Towards a network theory of the immune system. Ann Immunol (Paris) 1974; 125C: 373-389 